\title{
ESTIMATIVA DA BIOMASSA AÉREA E CARBONO DE Ilex microdonta EM FLORESTA ATLÂNTICA, PARANÁ, BRASIL
}

\author{
BIOMASS AND CARBON ESTIMATION OF Ilex microdonta IN ATLANTIC FOREST, \\ PARANÁ, BRAZIL
}
Bruno Palka Miranda ${ }^{1}$, Carlos Vellozo Roderjan², Paulo Cesar Botosso ${ }^{3}$,Tomaz Longhi-Santos4, Victor Hugo Ferreira Andrade ${ }^{5}$

1,2,4 Universidade Federal do Paraná, Curitiba, Paraná, Brasil - brunopalka@gmail.com, roderjan@ufpr.br\&tomazlonghi@gmail.com

${ }^{3}$ Embrapa Florestas, Colombo, Paraná, Brasil - paulo.botosso@embrapa.br

${ }^{5}$ Universidade Estadual do Centro Oeste, Irati, Paraná, Brasil - victorhugofandadre@gmail.com

RESUMO

\begin{abstract}
Conhecer o estoque de $\mathrm{C}$ fixado na vegetação é um dos importantes fatores para entender o balanço deste elemento nos ecossistemas e, para isso, o uso de modelos alométricos nestas estimativas é um dos meios de se obter esse tipo de dado, quando não é possível a amostragem direta de indivíduos nos ambientes. No âmbito da Floresta Ombrófila Densa no Paraná, os estudos de biomassa restringem-se aos patamares altitudinais inferiores da paisagem, inexistindo, portanto, estudos nos terços médio e superior das encostas da Serra do Mar. Neste contexto, esse trabalho teve por objetivo realizar uma estimativa dos valores de biomassa e carbono para llex microdonta, espécie de maior valor de importância nas comunidades altomontana da Serra do Mar, verificando se há diferenças entre os estoques em diferentes sítios avaliados. Foram amostradas 120 árvores em quatro diferentes sítios, sendo coletadas suas variáveis biométricas (diâmetros e alturas totais). Os estoques de biomassa e carbono foram estimados por meio de equações alométricas pantropicais e analisadas por sítio. Os dados foram submetidos ao teste de Kruskal-Wallis à um nível de probabilidade de $95 \%$. Não foi verificada diferenças estatísticas significativas entre as variáveis biométricas, nem entre as estimativas de biomassa e carbono entre os sítios. A estimativa de biomassa para $l$. microdonta nesses ambientes, computando a totalidade em superfície da Floresta Ombrófila Densa Altomontana, foi de 7,802 × $10^{4} \mathrm{Mg}$ de biomassa e 3,901 × $10^{4} \mathrm{Mg}$ de $\mathrm{C}$, dado este inédito para essa fitofisionomia da Floresta Ombrófila Densa no Paraná.
\end{abstract}

PALAVRAS-CHAVE: Mata nebular, Floresta Ombrófila Densa Altomontana, Aquifoliaceae, Modelos alométricos.

\section{ABSTRACT}

Knowing the $\mathrm{C}$ stock set in the vegetation is one of the key factors to understand the balance of this element in the ecosystems. For this, the use of allometric models in these estimations is one of the strategies to obtain this type of data, when it is not possible to direct sample the individuals in environments. Within the Dense Ombrophilous Forest in Paraná, biomass studies are restricted to the lower altitudinal levels of the landscape, therefore, there are no studies in the middle and upper parts of the Serra do Mar slopes. In this context, the objective of this work was to perform an estimation of biomass and carbon values for llex microdonta, a species of highest value of importance in the upper montane communities of Serra do Mar, verifying if there are differences between the stocks in different sites. A total of 120 trees were sampled at four different sites, and their biometric variables (diameters and total heights) were measured. The biomass and carbon stocks were estimated by allometric pantropical models and analyzed by site. The data were submitted to a Kruskal-Wallis test at a $95 \%$ probability level. There were no statistically significant differences between the biometric variables, nor between the biomass and carbon estimates between sites. The biomass estimation for I. microdonta in these environments, computing the totality of the Upper Montane Dense Ombrophylous Forest was $7.802 \times 10^{4} \mathrm{Mg}$ of biomass and 3,901 $\times 10^{4} \mathrm{Mg}$ of $\mathrm{C}$, an unprecedent data for this phytophysionomy of the Dense Ombrophylous Forest in Paraná.

KEYWORDS: Upper Montane Rain Forest, Tropical Montane Cloud Forests, Aquifoliaceae, Allometric models. 


\section{INTRODUÇÃO}

No âmbito do bioma Mata Atlântica no Paraná, a Floresta Ombrófila Densa Altomontana, popularmente conhecida como "mata nebular", ocupa as porções superiores da paisagem, acima de 1.200 metros sobre o nível do mar - m.s.n.m. (RODERJAN et al., 2002; IBGE, 2012). Ela é encontrada principalmente nos terços superiores das vertentes montanhosas da Serra do Mar, unidade fisiográfica que limita a planície litorânea à oeste. De acordo com um levantamento realizado pelo programa Pró-Atlântica (PARANÁ, 2005), a cobertura espacial dessa tipologia florestal alcança 5.760 ha, uma área consideravelmente restrita em comparação à superfície da Serra do Mar, que em sua totalidade possui, aproximadamente, $885 \mathrm{mil}$ ha.

Essa tipologia florestal tem uma ampla importância pelas suas funções ecológicas no ambiente: hidrológicas, por serem áreas de recarga hídrica e cabeceiras de drenagem das bacias hidrográficas associadas e na regulação dos fluxos hídricos a jusante (BRUIJNZEEL, 2001); geopedológicas, na estabilização mecânica das encostas, e pela enorme fixação de carbono nos solos orgânicos associados (SCHEER et al., 2011, 2013; VASHCHENKO \& BIONDI, 2013); e bióticas, pela ocorrência de espécies endêmicas e adaptadas às condições ambientais expostas nesses locais, tanto de flora quanto de fauna (HAMILTON et al., 1995).

Diversos são os estudos já realizados nesses ambientes, com ênfase na sua florística e fitossociologia e, dentre as espécies arbóreas ocorrentes nessa fitofisionomia, Ilex microdonta Reissek (Aquifoliaceae) prepondera, atingindo os maiores valores de importância nestas comunidades (RODERJAN, 1994; KOEHLER et al., 2002; SCHEER \& MOCOCHINSKI, 2009; SCHEER \& BLUM, 2011; VIEIRA et al., 2014).

Popularmente conhecida como caúna-da-serra, essa espécie pode alcançar até $8 \mathrm{~m}$ de altura, com folhas obovadas, coriáceas com margem serreada nos $2 / 3$ distais (EDWIN \& REITZ, 1967). Suas fenofases reprodutivas são encontradas de outubro (floração) até março (frutificação) (BROTTO et al., 2007; GROPPO, 2015). Espécie nãopioneira (WHITMORE, 1989) e endêmica do Brasil (GROPPO, 2015), ocorre principalmente nas matas nebulares das maiores elevações das encostas da Floresta Atlântica (EDWIN \& REITZ, 1967).

Entretanto, há ainda uma carência de estudos que indiquem, de fato, qual é a contribuição que as florestas altomontanas têm em relação aos estoques de biomassa e carbono, embora seu potencial de armazenamento e sequestro tenha sido enfatizado em estudos mais recentes (SPRACKLEN \& RIGHELATO, 2014; CUNI-SANCHEZ et al., 2017).

Britez et al. (2006) encontraram valores médios de 153 $\mathrm{Mg} \mathrm{C/ha} \mathrm{em} \mathrm{áreas} \mathrm{de} \mathrm{Floresta} \mathrm{Ombrófila} \mathrm{Densa,} \mathrm{em} \mathrm{pisos}$ altitudinais inferiores aos propostos por esse trabalho e considerando apenas a parte aérea da vegetação, e associa os valores encontrados principalmente à topografia local, com ênfase na profundidade dos solos e aporte de nutrientes. No solo, os estoques estimados de carbono nos ambientes altomontanos da Serra do Mar do Paraná (campos de altitude e florestas) ultrapassam a casa dos 1,7 $x 10^{6} \mathrm{Mg}$ (SCHEER et al., 2011).

$\mathrm{Na}$ conjuntura atual em termos de mudanças climáticas, é cada vez mais importante conhecer os estoques de biomassa e carbono atuais nos distintos ambientes (HAMILTON \& FRIESS, 2018). Ambientes com maior sensibilidade às mudanças climáticas, como a Floresta Ombrófila Densa Altomontana, podem estar sujeitos a uma dinâmica mais acelerada nas alterações dos estoques de carbono do que outros ambientes (HOUGHTON, 2005), e isso ainda pode ser agravado por sua baixa capacidade de resiliência (HAMILTON et al., 1995).

A metodologia de coleta de dados para estudos de biomassa geralmente envolve o abate de árvores, algo que é fortemente desencorajado e restritivo pela legislação ambiental vigente, uma vez que a maior parte da Serra do Mar paranaense está situada em Unidades de Conservação de Proteção Integral. Assim sendo, uma das alternativas é o uso de equações e modelos alométricos para estimar a biomassa e o carbono fixado. Este trabalho teve por objetivo realizar a estimativa de biomassa aérea e do carbono em uma espécie arbórea da Floresta Ombrófila Densa Altomontana em quatro montanhas da Serra do Mar paranaense, visando responder as seguintes perguntas: (1) São distintos os estoques de biomassa e carbono em cada uma das montanhas amostradas?; e (2) Qual é o atual estoque de biomassa aérea e carbono fixado para a espécie avaliada, no âmbito da Floresta Ombrófila Densa Altomontana?

\section{MATERIAL E MÉTODOS}

O trabalho foi desenvolvido em quatro montanhas da Serra do Mar paranaense (Figura 1), conforme denominação regional: Morro do Anhangava - AN: 1.430 m s.n.m.; Pico Capivari Grande - CP: 1.630 m s.n.m.; Pico Caratuva - CR: 1.850 m s.n.m.; e Morro do Sete - SE: 1.310 m s.n.m. Estas áreas localizam-se, respectivamente, nos 
municípios de Quatro Barras, Campina Grande do Sul, Antonina e Morretes, entre as coordenadas geográficas $25^{\circ} 12^{\prime} \mathrm{S}$ e $25^{\circ} 24^{\prime} \mathrm{S}$ e $48^{\circ} 52^{\prime} \mathrm{W}$ e $49^{\circ} 02^{\prime} \mathrm{W}$, aproximadamente.

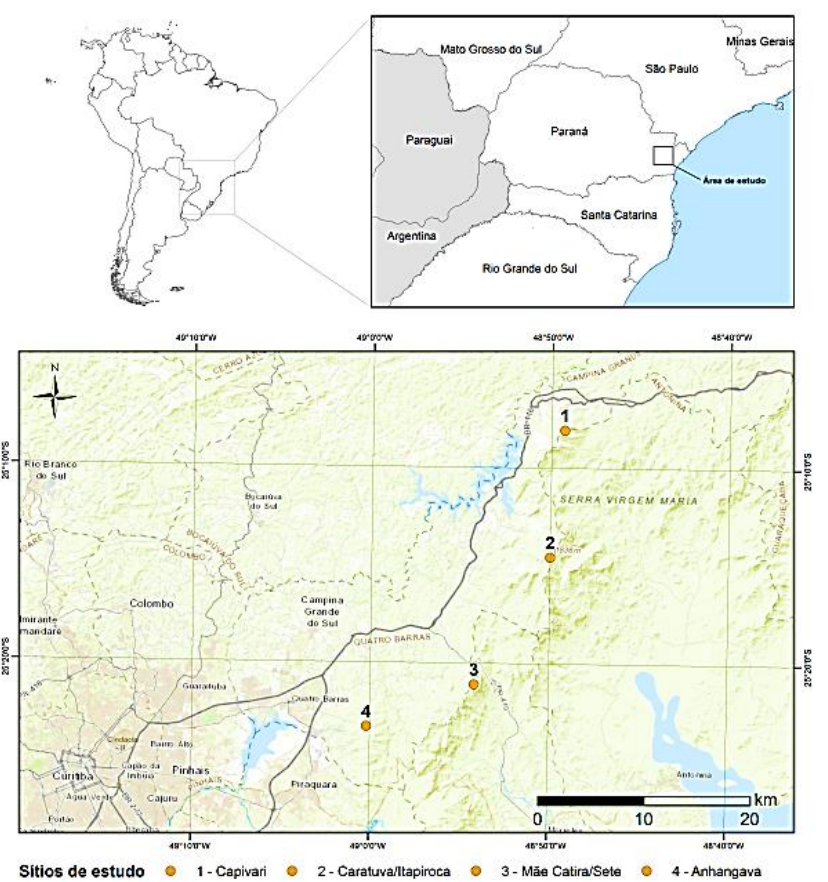

Figura 1. Localização das áreas de estudo, em destaque.

Foram coletados os dados de diâmetro a altura do peito (DAP)>10 cm e de altura total $(\mathrm{Ht})$ de 30 indivíduos arbóreos de I. microdonta por montanha, totalizando 120 árvores. Foram coletados também dados de latitude, longitude e altitude de amostragem, para cada indivíduo selecionado. Os limites de latitude e longitude das coletas em cada uma das montanhas foram utilizados para estimar uma área potencial de amostragem, que foi extrapolada para hectare (ha), a fim de estabelecer as comparações entre montanhas.

Para estimar a biomassa aérea dos indivíduos foi utilizado um modelo alométrico pantropical proposto por Chave et al. (2014), considerando o diâmetro, a altura total e a massa específica da espécie, conforme segue a equação:

$$
\mathrm{BA}=0,0673 \times\left(\rho \mathrm{D}^{2} \mathrm{Ht}\right)^{0,976}
$$

Em que: $\mathrm{BA}=$ biomassa aérea $(\mathrm{kg}) ; \rho=$ massa específica da madeira $\left(\mathrm{g} . \mathrm{cm}^{-1}\right) ; \mathrm{D}=$ diâmetro da árvore $(\mathrm{cm})$; $\mathrm{e}$ $\mathrm{Ht}=$ altura da árvore $(\mathrm{m})$.

Para o valor de massa específica da madeira de $I$. microdonta foi considerado o valor de $\rho=0,67 \mathrm{~g} \cdot \mathrm{cm}^{-1}$ (SOBOLESKI et al., 2017). Embora a mensuração direta da concentração de carbono na árvore não tenha sido realizada, estimou-se que o elemento representa em média $50 \%$ da matéria vegetal dos indivíduos avaliados (BRITEZ et al., 2006; WATZLAWICK et al., 2014).

Os valores de biomassa aérea foram estimados por montanha, por hectare e para o valor total em área de Floresta Ombrófila Densa Altomontana na Serra do Mar (área total $=5.760,7 \mathrm{ha}$ ), e comparados entre si pelo teste de Kruskal-Wallis ( $p<0,05)$, por meio do software Statgraphics Centurion XVI.II. Os gráficos de box-plot foram gerados pelo software $\mathrm{R}$.

\section{RESULTADOS E DISCUSSÃO}

\section{Dados de diâmetros e alturas totais}

Os dados de diâmetros e alturas médias encontrados foram de 16,65 cm e 5,15 m, respectivamente. Os valores médios para cada montanha são apresentados na Tabela 1. Esses valores condizem com levantamentos feitos nessa fitofisionomia no estado do Paraná (SCHEER, 2010; VIEIRA et al., 2014) e confirmam o porte reduzido das árvores, que denota ao ambiente a característica já citada por diversos autores, como uma "floresta anã" (HAMILTON et al., 1995).

Tabela 1. Estatísticas descritivas para o DAP e Altura Total nas montanhas avaliadas.

\begin{tabular}{|c|c|c|c|c|c|c|}
\hline & & AN & CP & CR & SE & Total \\
\hline \multirow{5}{*}{ 号 } & $\begin{array}{l}\text { Méd. } \\
\text { (DP) }\end{array}$ & $\begin{array}{c}16,71 a \pm \\
4,23\end{array}$ & $\begin{array}{c}17,40 a \pm \\
5,80\end{array}$ & $\begin{array}{c}15,63 a \pm \\
3,82\end{array}$ & $\begin{array}{c}16,88 a \pm \\
4,32\end{array}$ & $\begin{array}{c}16,65 \pm \\
4,60\end{array}$ \\
\hline & CV & 0,25 & 0,33 & 0,24 & 0,26 & 0,28 \\
\hline & Mín. & 11,78 & 10,50 & 10,50 & 10,82 & 10,50 \\
\hline & Máx. & 28,33 & 35,65 & 28,81 & 30,88 & 35,65 \\
\hline & Amp. & 16,55 & 25,15 & 18,30 & 20,05 & 25,15 \\
\hline \multirow{5}{*}{ 蒫 } & $\begin{array}{l}\text { Méd. } \\
\text { (DP) }\end{array}$ & $\begin{array}{c}5,52 a \pm \\
0,80\end{array}$ & $\begin{array}{c}5,08 a b \pm \\
0,90\end{array}$ & $\begin{array}{c}5,31 b c \pm \\
0,84\end{array}$ & $\begin{array}{c}4,69 c \pm \\
0,76\end{array}$ & $\begin{array}{c}5,15 \pm \\
0,87\end{array}$ \\
\hline & CV & 0,14 & 0,18 & 0,16 & 0,16 & 0,17 \\
\hline & Mín. & 4,00 & 3,50 & 3,50 & 3,50 & 3,50 \\
\hline & Máx. & 7,00 & 6,50 & 6,50 & 6,50 & 7,00 \\
\hline & Amp. & 3,00 & 3,00 & 3,00 & 3,00 & 3,50 \\
\hline
\end{tabular}

Média (DP) = valores em $\mathrm{cm}$ e $\mathrm{m}$, para DAP e Ht, respectivamente ( \pm desvio padrão); CV = Coeficiente de Variação em \%; Amp. = amplitude dos dados (cm, para o DAP, e $\mathrm{m}$ para $\mathrm{Ht}$ ).

Para I. microdonta, a variável de diâmetro não obteve diferença estatística significativa entre as montanhas ( $p$ valor $=0,61)$. Entretanto, para a variável altura, as montanhas diferiram entre si ( $p$-valor $=0,001)$. Essa diferença poderia ser justificada pelos distintos níveis de declividade e forma de rampa das vertentes, que acabam por restringir a profundidade dos solos e a estabilização 
mecânica das raízes das árvores, em que trechos mais declivosos das florestas sobre encostas divergentes tenderiam a reduzir as alturas das árvores lá estabelecidas (VIEIRA, 2016).

\section{Biomassa e carbono}

Devido ao porte dos indivíduos, os valores de biomassa aérea (em $\mathrm{kg}$ ) foram perceptivelmente inferiores aos relatados para outras porções da Floresta Atlântica (BRITEZ et al., 2006), ainda que somente para uma espécie. Entretanto, os valores médios por montanha não foram significativamente distintos ( $p$-valor $=0,79$ ) entre si, denotando que o estoque de biomassa nos indivíduos amostrados é similar entre as montanhas (Figura 2).

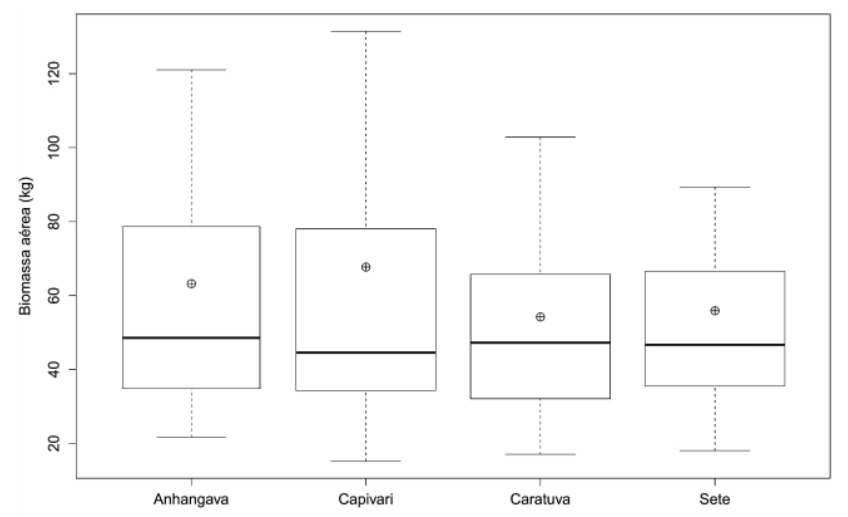

Figura 2. Box-plot dos valores médios de biomassa das árvores individuais, entre os diferentes sítios.

Os valores médios por hectare das montanhas foram de 1,023 Mg.ha-1 (AN), 1,614 Mg.ha-1 (CP), 1,421 Mg.ha-1 (CR) e 1,824 Mg.ha-1 (SE), obtendo como valor médio 1,4705 Mg.ha-1. Em relação ao carbono, os estoques atuais do elemento correspondem a $50 \%$ dos valores de biomassa, obtendo 0,5115 Mg.ha-1 C (AN), 0,807 Mg.ha-1 C (CP), 0,7105 Mg.ha-1 C (CR) e 0,912 Mg.ha-1 C (SE), obtendo como valor médio 0,736 Mg.ha-1 C. Esses valores diferem substancialmente das estimativas para outras fitofisionomias dentro da mesma unidade fitoecológica (MARCHIORI et al., 2016). Entretanto deve-se levar em consideração que esses valores são de apenas uma espécie arbórea, ainda que esta seja a de maior valor de importância nessas comunidades.

Como se trata de uma única espécie dessas florestas, a massa específica da madeira - associada aos diâmetros e alturas similares, permite inferir que o comportamento do acúmulo de biomassa e de $C$ deva ser também similar entre os diferentes sítios avaliados. Assim, ao extrapolar o valor médio dos dados amostrados para a área total da fisionomia no Estado do Paraná, obtêm-se uma estimativa

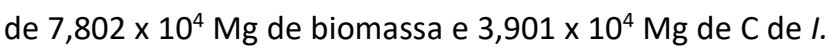
microdonta na Serra do Mar do Paraná.

As estratégias de alocação de recursos provenientes da fotossíntese nesses ambientes são diferenciadas, uma vez que estão sujeitas às constantes alterações climáticas, em termos de radiação solar pela presença constante das nuvens no sistema, input hídrico (tanto vertical quanto horizontal) e variações de temperatura, fortalecidas pelo gradiente adiabático e altitudinal, entre outros (AIBA \& KITAYAMA, 1999; RAICH et al., 2006; MOSER et al., 2007). Assim, o crescimento de 1. microdonta e das demais espécies formadoras dessas florestas é reduzido, comparado a outros pisos altitudinais (ALVES et al., 2010; COSMO, 2012; SHIMAMOTO et al., 2016) e acaba por ser fator determinante na biomassa e nos teores de $C$ encontrados. Além das taxas de incremento, a idade dos indivíduos também é fator preponderante na variação e acúmulo da biomassa nas árvores (SHIMAMOTO, 2012).

Considerada uma espécie pioneira a secundária inicial (MANTOVANI et al., 2005; CHAMI et al., 2011), os teores de $C$ na biomassa de $I$. microdonta levam em conta os diferentes compartimentos de alocação do $C$ (fuste, raízes, folhas, galhos) e sua própria arquitetura pode ter influência nos resultados esperados. Entretanto, modelos alométricos desenvolvidos para a estimativa de biomassa e consequentemente $C$ nas árvores apresentam redução dos erros e menor AIC (CHAVE et al., 2005; CHAVE et al., 2014) baseados nos diâmetros dos fustes e altura da árvore, variáveis em que a concentração tende a ser mais elevada em comparação aos outros compartimentos (CHAVE et al., 2005; ARIAS et al., 2011).

\section{CONCLUSÕES}

Os resultados permitem concluir que não foi verificada diferença estatística significativa das estimativas de biomassa e carbono entre as montanhas avaliadas.

Ainda, foi possível estimar a contribuição de llex microdonta no estoque atual de biomassa e carbono, de

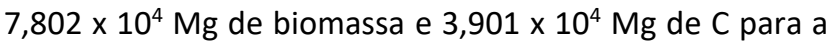
Floresta Ombrófila Densa Altomontana no Paraná.

\section{AGRADECIMENTOS}

O presente trabalho foi realizado com apoio da Coordenação de Aperfeiçoamento de Pessoal de Nível Superior - Brasil (CAPES) - Código de Financiamento 001. 


\section{REFERÊNCIAS}

AIBA, S.; KITAYAMA, K. Structure, composition and species diversity in an altitude-substrate matrix of rain forest tree communities on Mount Kinabalu, Borneo. Plant Ecology, v.140, n.2, p.139-157, 1999.

ALVES, L.F. et al. Forest structure and live aboveground biomass variation along an elevational gradient of tropical Atlantic moist forest (Brazil). Forest Ecology and Management, v.260, n.5, p.679-691, 2010.

ARIAS, D. et al. Productivity, aboveground biomass, nutrient uptake and carbon content in fast-growing tree plantations of native and introduced species in the Southern Region of Costa Rica. Biomass and Bioenergy, v.35, n.5, p.1779-1788, 2011.

BRITEZ, R.M. et al. Estoque e incremento de carbono em florestas e povoamentos de espécies arbóreas com ênfase na Floresta Atlântica no sul do Brasil. Colombo: Embrapa Florestas, 2006.

BROTTO, M.L. et al. Flórula do Morro dos Perdidos, Serra de Araçatuba, Paraná, Brasil: Aquifoliaceae. Estudos de Biologia, v.29, n.67, p.129-135, 2007.

BRUIJNZEEL, L.A. Hydrology of tropical montane cloud forests: A Reassessment. Land Use and Water Resources Research, v.1, p.118, 2001.

CHAMI, L.B. et al. Mecanismos de regeneração natural em diferentes ambientes de remanescente de Floresta Ombrófila Mista, São Francisco de Paula, RS. Ciência Rural, v.41, n.2, p.251259, 2011.

CHAVE, J. et al. Tree allometry and improved estimation of carbon stocks and balance in tropical forests. Oecologia, v.145, p.87-99, 2005.

CHAVE, J. et al. Improved allometric models to estimate the aboveground biomass of tropical trees. Global Change Biology, v.20, n.10, p.3177-3190, 2014

COSMO, N.L. Ecologia do lenho de 19 espécies nativas do estado do Paraná. 2012. 80p. (Tese de Doutorado).

CUNI-SANCHEZ, A. et al. New insights on above ground biomass and forest attributes in tropical montane forests. Forest Ecology and Management, v.399, p.235-246, 2017.

EDWIN, G.; REITZ, P.R. Aquifoliáceas. Flora ilustrada catarinense. Itajaí: Herbário Barbosa Rodrigues, 1967.

GROPPO, M. Aquifoliaceae. Lista de espécies da flora do Brasil, Rio de Janeiro: Jardim Botânico do Rio de Janeiro, 2015.

HAMILTON, L.S. et al. Tropical montane cloud forests. New York: Springer-Verlag, 1995.

HAMILTON, S.E.; FRIESS, D.A. Global carbon stocks and potential emissions due to mangrove deforestation from 2000 to 2012. Nature Climate Change, v.8, n.3, p.240-244, 2018.

HOUGHTON, R.A. Aboveground forest biomass and the global carbon balance. Global Change Biology, v.11, n.6, p.945-958, 2005.

IBGE. Manual técnico da vegetação brasileira. Rio de Janeiro, 2012.

KOEHLER, A. et al. Floresta Ombrófila Densa Altomontana: aspectos florísticos e estruturais de diferentes trechos na Serra do Mar, PR. Ciência Florestal, v.12, n.2, p.27-39, 2002.

MANTOVANI, M. et al. Diversidade de espécies e estrutura sucessional de uma formação secundária da floresta ombrófila densa. Scientia Forestalis, n.67, p.14-26, 2005.

MARCHIORI, N.M. et al. Tree Community Composition and Aboveground Biomass in a Secondary Atlantic Forest, Serra Do Mar State Park, São Paulo, Brazil. Cerne, v.22, n.4, p.501-514, 2016.

MOSER, G. et al. Altitudinal change in LAl and stand leaf biomass in tropical montane forests: A transect study in ecuador and a pan-tropical meta-analysis. Ecosystems, v.10, n.6, p.924-935, 2007.

PARANÁ. Atlas da Floresta Atlântica no Paraná. Curitiba, 2005.

RAICH, J.W. et al. Temperature Influences Carbon Accumulation in Moist Tropical Forests. Ecology, v.87, n.1, p.76-87, 2006.

RODERJAN, C.V. O gradiente da Floresta Ombrófila Densa no Morro Anhangava, Quatro Barras, PR - Aspectos climáticos, pedológicos e fitossociológicos. 1994. 130p. (Tese de Doutorado).

RODERJAN, C.V. et al. As unidades fitogeográficas do estado do Paraná, Brasil. Ciência \& Ambiente, v.1, n.24, p.1-12, 2002.

SCHEER, M.B. Ambientes altomontanos no Paraná: florística vascular, estrutura arbórea, relações pedológicas e datações por 14C. 2010. 153p. (Tese de Doutorado).

SCHEER, M.B.; BLUM, C.T. Arboreal diversity of the Atlantic Forest of Southern Brazil: From the beach ridges to the Paraná River. The dynamical processes of biodiversity - case studies of evolution and spatial distribution, 2011.

SCHEER, M.B. et al. Funcionalidades ambientais de solos altomontanos na Serra da Igreja, Paraná. Revista Brasileira de Ciencia do Solo, v.35, n.4, p.1113-1126, 2011.

SCHEER, M.B. et al. Carbon and water in upper montane soils and their influences on vegetation in Southern Brazil. ISRN Soil Science, v.2013, p.1-12, 2013.

SCHEER, M.B.; MOCOCHINSKI, A.Y. Florística vascular da floresta ombrófila densa altomontana de quatro serras no Paraná Florística vascular da Floresta Ombrófila Densa Altomontana de quatro serras no Paran. Biota Neotropica, v.9, n.2, p.51-70, 2009.

SHIMAMOTO, C.Y. Estimativa do crescimento e acúmulo de biomassa em espécies arbóreas, como subsídio a projetos de restauração da Mata Atlântica. 2012. 52p. (Tese de Doutorado).

SHIMAMOTO, C.Y. et al. Stem growth rhythms in trees of a 
tropical rainforest in Southern Brazil. Trees - Structure and Function, v.30, n.1, p.99-111, 2016.

SOBOLESKI, V.F. et al. Variação de atributos funcionais do componente arbóreo em função de gradientes edáficos em uma floresta nebular no sul do Brasil. Rodriguésia, v.68, n.2, p.291300, 2017.

SPRACKLEN, D.V.; RIGHELATO, R. Tropical montane forests are a larger than expected global carbon store. Biogeosciences, v.11, n.10, p.2741-2754, 2014.

VASHCHENKO, Y.; BIONDI, D. Percepção da erosão pelos visitantes nas trilhas do Parque Estadual do Pico Marumbi, PR. Revista Brasileirade Ciencias Agrarias, v.8, n.1, p.108-118, 2013.

VIEIRA, R.S. Influência de feições geomórficas sobre os padrões pedológicos, florísticos e estruturais de florestas altomontanas na Serra do Mar do Paraná - Brasil. 2016. 83p. (Dissertação de Mestrado).

VIEIRA, R.S. et al. Floresta ombrófila densa altomontana na Serra do Capivari , Campina Grande do Sul , Paraná. Floresta, v.44, n.4, p.565-576, 2014.

WATZLAWICK, L.F. et al. Teores de carbono em espécies da Floresta Ombrófila Mista e efeito do grupo ecológico. Cerne, v.20, n.4, p.613-620, 2014.

WHITMORE, T.C. Canopy gaps and the two major groups of forest trees. Ecological Society of America, v.70, n.3, p.536-538, 1989. 